[0212-7199 (2007) 24: 1; pp 27-30] ANALES DE MEDICINA INTERNA Copyright (C) 2007 ARAN EDICIONES, S.L.

AN. MED. INTERNA (Madrid) Vol. 24, N. ${ }^{\circ} 1$, pp. $27-30,2007$

\title{
Espondilodiscitis dorsal neumocócica y derrame pleural. Aportación de un caso y revisión de la literatura
}

\author{
N. GÓMEZ RODRÍGUEZ, O. DURÁN MUÑOZ1 \\ Servicios de Reumatología y ${ }^{\prime}$ Medicina Interna. Hospital POVISA. Vigo. Pontevedra
}

\begin{abstract}
RESUMEN
En los adultos, las osteomielitis producidas por Streptococcus pneumoniae son muy infrecuentes y, excepto casos aislados, apenas hay referencias de osteomielitis vertebrales por este patógeno. Describimos una paciente de 73 años sin compromiso inmune, que fue ingresada por fiebre, dolor abdominal y dolor dorsal inflamatorio cuyo estudio llevó al diagnóstico de derrame pleural derecho y espondilodiscitis dorsal (D10D11) en la que se aisló por punción aspirativa Streptococcus pneumoniae. Se revisa la literatura médica sobre espondilodiscitis neumocócicas entre 1970 y 2005.
\end{abstract}

PALABRAS CLAVE: Streptococcus pneumoniae. Osteomielitis vertebral. Espondilodiscitis infecciosa. Derrame pleural.
PNEUMOCOCCAL SPONDYLODISCITIS AND PLEURAL EFFUSION. REPORT OF A CASE AND LITERATURE REVIEW

Gómez Rodríguez N, Durán Muñoz O. Espondilodiscitis dorsal neumocócica y derrame pleural. Aportación de un caso y revisión de la literatura. An Med Interna (Madrid) 2007; 24: 27-30.

\section{INTRODUCCIÓN}

Streptococcus pneumoniae es el principal agente etiológico de las neumonías en pacientes adultos y también es una causa relevante de meningitis (1). Por el contrario, las artritis y osteomielitis se consideran infrecuentes, ya que, en conjunto, representan menos del $3 \%$ de las infecciones por este patógeno. No obstante, como refleja la revisión de Ispahani (2), las infecciones osteoarticulares neumocócicas podrían estar aumentando. La localización vertebral de la osteomielitis neumocócica en adultos es un hecho excepcional, sobre todo cuando no existen enfermedades sistémicas ni compromiso inmune (3). Por este motivo hemos decidido aportar un nuevo caso de espondilodiscitis neumocócica en una mujer de 73 años, sin factores predisponentes generales, en quien coexistía un derrame pleural derecho y cuya evolución con tratamiento conservador fue favorable. Asimismo, revisamos la literatura nacional e internacional mediante Medline-Embase-plus y Ovid entre 1970 y 2005.

\begin{abstract}
Streptococcus pneumoniae osteomyelitis in adults appears to be a rare event and, apart from isolated case reports, vertebral osteomyelitis due to this pathogen has rarely been reported. We described a immunocompetent 73-year-old woman who was admitted for fever, abdominal and inflammatory back pain. She was diagnosed of T10-T11 spondylodiscitis and right pleural effusion based on computed tomography and magnetic resonance imaging. Streptococcus pneumoniae was isolated from an aspirate of the vertebral foccus. The medical literature for other cases of pneumococcal spondylodiscitis was conducted from 1970 to 2005.
\end{abstract}

KEY WORDS: Streptococcus pneumoniae. Vertebral osteomyelitis. Infectious spondylodiscitis. Pleural effusion.

\footnotetext{
Trabajo aceptado: 18 de julio de 2006
} 
o negativos: urea, creatinina, bilirrubina, transaminasas, gammaglutamiltranspeptidasa, sodio, potasio, cloro, calcio, fósforo, creatinfosfoquinasa, amilasa, lácticodehidrogenasa $(\mathrm{LDH})$, proteínograma, dosificación de inmunoglobulinas, alfa-fetoproteína, antígenos CA 19.9 y CA 125, así como el análisis general de orina. La VSG y la proteína $\mathrm{C}$ reactiva estaban elevadas, con valores de $85 \mathrm{~mm} / 1^{\mathrm{a}} \mathrm{h}$ y de $133 \mathrm{mg} / \mathrm{L}$ (normal < $5 \mathrm{mg} / \mathrm{L}$ ), respectivamente. La intradermorreacción de Mantoux (10 UI RT-23) fue positiva, con una induración de $25 \mathrm{~mm}$. La radiografía de tórax mostraba una imagen compatible con atelectasia del lóbulo inferior derecho en el contexto de un derrame pleural ipsilateral. No había signos sugestivos de adenopatías ni alteraciones en la silueta cardiopericárdica. Una TAC toracoabdominal confirmó la existencia de un derrame pleural derecho (Fig. 1) e identificó prominentes cambios degenerativos a lo largo del raquis dorsolumbar pero, sobre todo, erosiones en los platillos vertebrales adyacentes al espacio discal D10-D11. Una resonancia lumbar mostró hiposeñal en las secuencias ponderadas en T1 e hiperseñal en las ponderadas en $\mathrm{T} 2$ en dichas vértebras y su correspondiente disco, con alteraciones morfológicas típicas de espondilodiscitis infecciosa D10D11 (Fig. 2). Tres hemocultivos seriados fueron negativos. En las muestras obtenidas por punción aspirativa del espacio D10-D11 se observaron cadenas de cocos grampositivos que posteriormente se recuperaron y tipificaron como Streptococcus pneumoniae sensible a penicilina. El análisis del líquido pleural mostró pH: 7,55; leucocitos: 8,4 x 109 / L (58\% neutrófilos, $26 \%$ eosinófilos, $16 \%$ linfocitos), proteínas: $48 \mathrm{~g} / \mathrm{L}$ (cociente respecto a las proteínas séricas: 0,65), glucosa: $125 \mathrm{mg} / \mathrm{dl}$, ADA: 25,92 UI/ ml, LDH: 362 U/L (Cociente LDH pleural / LDH sérica: 0,8). Tanto la tinción de auramina-rodamina como el cultivo en medio LöwensteinJensen del líquido pleural fueron negativos y la citología no evidenció células neoplásicas.

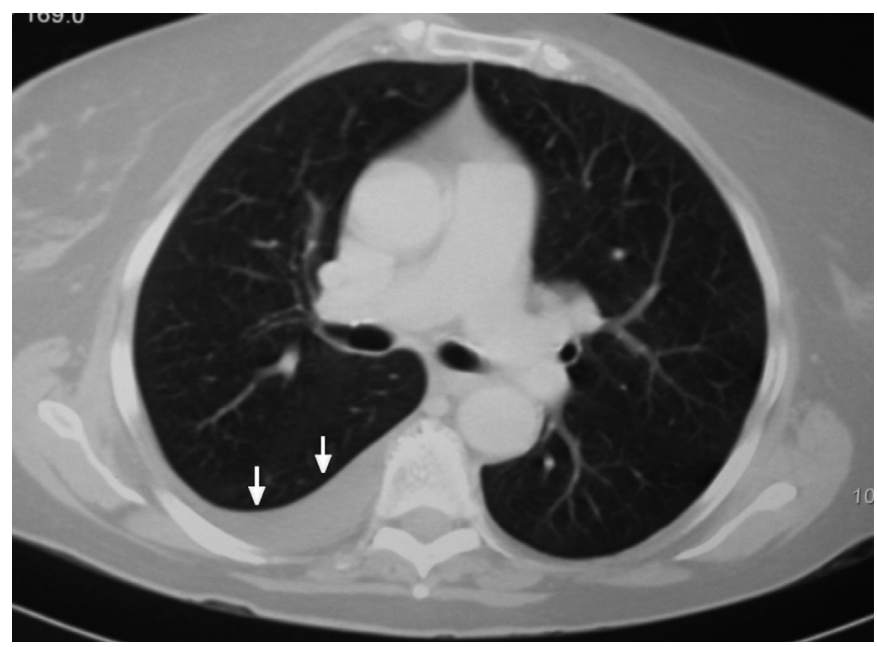

Fig. 1. Corte de la tomografía computarizada realizada a las 48 horas del ingreso de la paciente en el que puede apreciarse el derrame pleural derecho.

La paciente fue tratada inicialmente por vía intravenosa con la asociación de amoxicilina + clavulánico (1 g / $200 \mathrm{mg}$, cada 8 horas). Transcurridos 21 días se pasó a la vía oral (875 / $125 \mathrm{mg}$, cada 8 horas) hasta completar 6 semanas. La evolución fue favorable y pudo iniciar la deambulación con corsé dorsolumbar a partir de la cuarta semana. Un mes después de finalizada la antibioterapia, una TAC torácica de control todavía mostraba un discreto derrame pleural, pero la enferma sólo aquejaba dorsalgias mecánicas de escasa intensidad, la VSG había disminuido a $21 \mathrm{~mm} / 1^{\text {a }} \mathrm{h}$ y la PCR era de 2,4 mg/L. El seguimiento ambulatorio se prolongó tres años más, durante los que la evolución fue favorable y se formó un bloque vertebral D10-D11.

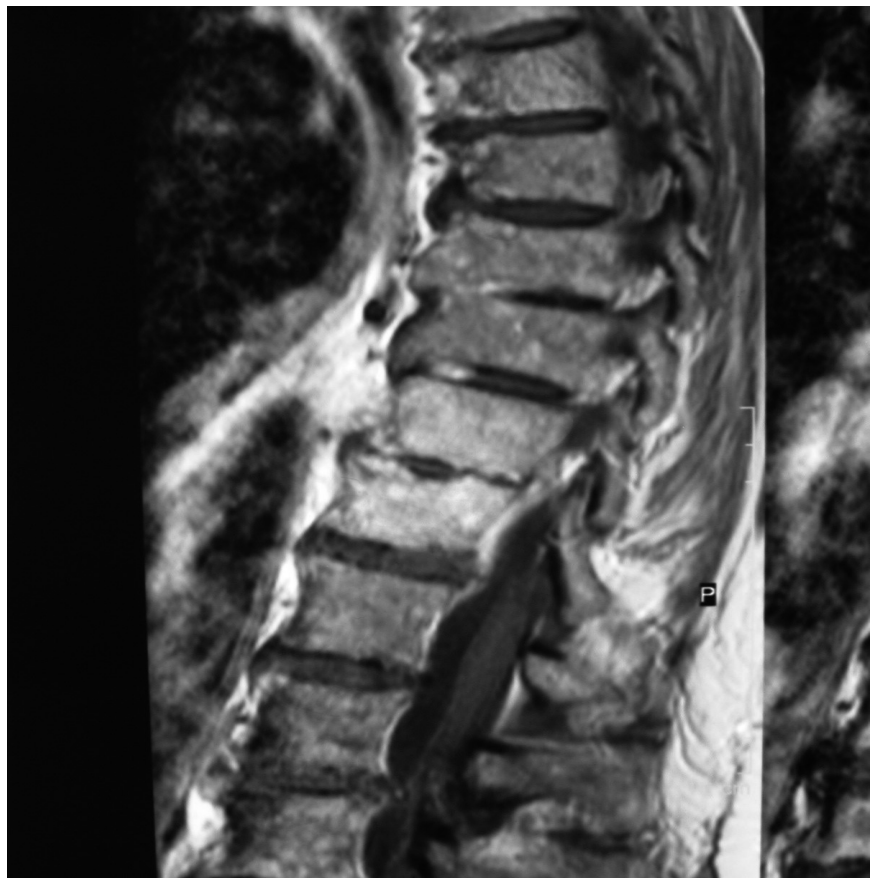

Fig. 2. Corte sagital de resonancia magnética ponderado en T2. Puede apreciarse, además de cambios degenerativos generalizados en las vértebras dorsales y lumbares, una hiperseñal de los cuerpos y espacio discal D10-D11, cuya altura está disminuida.

\section{DISCUSIÓN}

Streptococcus pneumoniae causa menos del $10 \%$ de las espondilodiscitis infecciosas y se ha estimado que sólo ocho de cada mil bacteriemias neumocócicas se complican con focos de osteomielitis vertebral (3). En la revisión de la literatura hemos localizado otros 38 casos de espondilodiscitis neumocócicas (2-29), la mayoría en adultos (33 / 38; 87\%), con predominio de los varones $(61 \%)$ y con una mediana de edad de 51 años (rango: 6 meses - 79 años). La principal localización fue lumbar (63\%), seguida de la cervical $(25 \%)$ y dorsal (22\%). Aunque en siete (18\%) estuvieron implicados varios segmentos del raquis, sólo se objetivaron lesiones politópicas en dos (5\%). El tiempo transcurrido entre el inicio de los síntomas osciló entre sólo dos días y cinco meses, con una media de cuatro semanas.

Las vías respiratorias son la principal puerta de entrada para Streptococcus pneumoniae y se documentaron infecciones en esta localización en 13 pacientes (34\%), aunque sólo cuatro de ellas fueron neumonías (2-29). El principal factor predisponente en los adultos fue el alcoholismo (18\%), seguido de la diabetes mellitus (12\%), pero en el $62 \%$ de los casos no se identificó enfermedad sistémica o compromiso inmune. Si bien los pacientes afectados por neoplasias hematológicas y tumores sólidos son más susceptibles a las infecciones bacterianas, entre ellas a las producidas por Streptococcus pneumoniae (30), no identificamos este grupo de procesos entre los afectados por espondilodiscitis neumocócicas (2-29). En el caso aquí descrito no se recogieron síntomas que sugiriesen una reciente infección respiratoria y tampoco factores predisponentes sistémicos. Por otra parte, aunque al ingreso la glucemia estaba aumentada (123 
$\mathrm{mg} / \mathrm{dl}$ ), sus valores permanecieron dentro de la normalidad durante los tres años de seguimiento en consultas externas, periodo durante el cual tampoco hubo evidencia de neoplasia o disfunción inmune. No obstante, los traumatismos directos sobre el raquis y las lesiones previas como fracturas vertebrales y la espondiloartrosis, como la que presentaba nuestra paciente, pueden actuar como factor focalizador, favoreciendo la decantación de patógenos en caso de bacteriemia $(31,32)$.

En todas las espondilodiscitis neumocócicas en las que se determinó, la VSG en la primera hora estuvo elevada y su valor superó los $50 \mathrm{~mm}$ en el $87 \%$ de los enfermos. Hubo leucocitosis en el $72 \%$ de los enfermos y la rentabilidad de los hemocultivos fue del $52 \%$.

El derrame pleural es un hallazgo infrecuente en la espondilodiscitis infecciosa dorsal (33-39) pero puede constituir su forma de presentación (37). Como en nuestro caso, en la mayoría de los enfermos, el derrame es estéril y estaría inducido por un mecanismo irritativo (exudativo), debido a la proximidad del foco de osteomielitis. Sin embargo, cuando existe un empiema pulmonar, la espondilodiscitis podría ser secundaria a la infección pleural $(38,39)$. En la literatura revisada (2-29) no hemos identificado otros casos de derrame pleural asociado a espondilodiscitis por Streptococcus pneumoniae, pero destacamos complicaciones tales como ocho abscesos epidurales adyacentes al foco de osteomielitis, todos con semiología de compromiso mielorradicular, cinco meningitis, dos aneurismas micóticos $(25,29)$ y una mediastinitis (4). Un 2-6\% de los pacientes con endocarditis infecciosa presentan focos de espondilodiscitis y la sintomatología derivada de la afectación vertebral puede constituir la primera manifestación clínica (31). Entre los enfermos con espondilodiscitis neumocócica, cinco (13\%) presentaban endocarditis $(3,8,17)$, tres de ellos con meningitis concomitante.

Además de la oportuna antibioterapia, el $45 \%$ de los enfermos requirieron tratamiento quirúrgico. Aunque en la mayoría de las series de espondilodiscitis piógenas (40-45), la mortalidad no supera el 5\%, ésta alcanzó el $25 \%$ entre los enfermos con espondilodiscitis por Streptococcus pneumoniae (2-29), lo que refleja la potencial virulencia del microorganismo y la elevada frecuencia con la que coexisten otros focos de infección, particularmente meningitis y endocarditis. No se observaron diferencias significativas en la evolución de los casos producidos por cepas de neumococo resistente a la penicilina frente a las susceptibles a dicho antibiótico (3), situación similar a la objetivada en las infecciones respiratorias $(1,30)$.

En resumen, la espondilodiscitis es una complicación excepcional de la bacteriemia por Streptococcus pneumoniae, que coexiste con frecuencia con otros focos de infección, por este microorganismo y, cuando ello ocurre, tiene peor pronóstico.

\section{Bibliografía}

1. Musher D, Alexandraki I, Graviss E, Yanbeiy N, Ahmad E, Inderias L et al. Bacteriemic and nonbacteriemic pheumococcal pneumonia: A prospective study. Medicine (Balt) 2000; 79: 210-221.

2. Ispahani P, Weston V C, Turner D P J, Donald F E. Septic arthritis due to streptococcus pneumoniae in Nottingam, United Kingdom, 1985-1998. Clin Infect Dis 1999; 29: 1.450-1.454.

3. Turner D P, Weston V C, Ispahani P. Streptococcus pneumoniae spinal infection in Nottingham, United Kingdom: not a rare event. Clin Infect Dis 1999; 28: 873-881.

4. Klein H M. Acute osteomyelitis of the vertebrae. Arch Surg 1933; 26: 169-195.

5. Browder J, Meyers R. Infectious of the spinal epidural space: an aspect of vertebral osteomyelitis. Am J Surg 1937; 37: 4-26

6. Griffiths H E D, Jones D M. Pyogenic infections of the spine: a review of twenty-eigth cases. J Bone Joint Surg 1971 (Br); 53: 383-391

7. Enberg R N, Kaplan R J. Spinal epidural abscess in chindren: early diagnosis and inmediate surgical drainage is essential to forestall paralysis. Clin Pediatr (Phila) 1974; 13: 247-248, 251-253.

8. Kaufman D M, Kaplan RJ G, Litman N. Infectious agents in spinal epidural abscesses. Neurology 1980; 30: 844-850.

9. Schleiter G, Gantz N M. Vertebral ostemomyelitis secondary to streptococcus pneumoniae: a pathophysiologic understanding. Diagn Microbiol Infect Dis 1986; 5: 77-80.

10. Peterson J A, Paris P, Williams A C. Acute epidural abscess. Am J Emerg Med 1987; 5: 287-290.

11. Gelfand M S, Miller J H. Pneumococcal vertebral osteomyelitis in an adult. South Med J 1987; 80: 534-535

12. Malleson P N, Gross K R, Hardyment A, Petty R E. Pneumococcal vertebral ostemyelitis presenting with an aseptic knee effusion in a child. Clin Exp Rheumatol 1988; 6: 325-328.

13. Marks W A, Bodensteiner J B. Anterior cervical epidural abscess with Streptococcus pneumoniae in an infant. J Child Neurol 1988; 3: 25-29.

14. Clark R, Carlisle J T, Valainis G T. Streptococcus pneumoniae endocar- ditis presenting as an epidural abscess. Rev Infect Dis 1989; 11: 338-340. 15. Briones H, Rodríguez-Valverde V, Álvarez B, Peiró E, Sánchez Andrade S. Espondilodiscitis piógena. Estudio de nueve pacientes. Rev Clin Esp 1987; 180: 432-434.

16. Gelfand M S, Miller J H. Penicillin-resistant pneumococcal vertebral osteomyelitis. Clin Infect Dis 1992; 15: 746-747.

17. García A M, Uribeechevarría E, Urcelay G, Yerobi J. Endocarditis subaguda y espondilodiscitis por Streptococcus pneumoniae. Med Clin (Barc) 1994; 102: 797-798.

18. Kutas L M. Duggan J M, Kauffman C A. Pneumococcal vertebral osteomyelitis. Clin Infec Dis 1995; 20: 286-290.

19. Escriva B, Cesar-Blázquez J, Llavero J M, Tovar J. Espondilodiscitis por Streptococcus pneumoniae. Enf Infecc Microbiol Clin 1996; 14: 198199.

20. Arraz J A, Sole N, Sánchez A, Gómez P. Penicillin-intermediate-resistant pneumococcal spondylodiscitis. Diag Microbiol Infec Dis 1996; 26: $137-$ 139.

21. Touchard P, Choue P Y, Fulping J, Jeandel P. [HIV infection manifesting as a pneumococcal spondylodiscitis]. Medecine Tropicale 1996; 56: 275278.

22. Chemlal K, Trouillet J L, Carbon C, Yeni P. Vertebral osteomyelitis and meningitis due to a penicillin-resistant pneumococcal strain. Eur J Clin Microbiol Infect Dis 1996; 15: 893-895.

23. Babinchat T J, Riley D K, Rotheram E B. Pyogenic vertebral osteomyelitis of the posterior elements. Clin Infect Dis 1997; 25: 221-224.

24. Antony S J. Multidrug-resistan Pneumococcus causing vertebral osteomyelitis. J Nat Med Assoc 1997; 89: 634-635.

25. Naktin J, De Simone J. Lumbar vertebral osteomyelitis with mycotic abdominal aortic aneurysm caused by highly penicillin-resistant Streptococcus pneumoniae. J Clin Microbiol 1999; 37: 4.198-4.200.

26. Belzunegui J, Intxausti J J, De Dios J R, Del Val N, Rodríguez-Valverde $\mathrm{V}$, González $\mathrm{C}$ et al. Haematogenous vertebral osteomyelitis in the elderly. Clin Rheumatol 2000; 19: 344-347. 
27. Poyanli A, Poyanli O, Akan K, Sencer S. Pneumococcal vertebral osteomyelitis: a unique case with a atypical clinical course. Spine 2001 2.397-2.399

28. Nolla J M, Ariza J, Gómez-Vaquero C, Fiter J, Bermejo J, Valverde J et al. Spontaneous pyogenic vertebral osteomyelitis in nondrug users. Semin Arthritis Rheum 2002; 31: 271-278.

29. Englert C, Aebert H, Lenhart M, Solleder A, Nerlich M, Neumann C. Thoracic spondylitis from a mycotic (Streptococcus pneumoniae) aortic aneurysm. Spine 2004; 29: E-373-E365.

30. Kumashi P, Girgawy E, Tarrand J J, Rolston K V, Raad I I, Safdar A. Streptococcus pneumoniae bacteremia in patients with cancer. Medicine (Balt) 2005; 84: 303-312.

31. Fiter J, Gómez C, Miquel J. Espondilodiscitis infecciosa. Perspectiva actual de un viejo problema. Rev Esp Reumatol 1999; 26: 91-99.

32. Chan E D, Kong P M, Fennelly K, Dwyer A D, Iseman M D. Vertebral osteomyelitis due to infection with nontuberculous Mycobacterium Species after blunt trauma to the back: 3 examples of the principle of locus minoris resistentiae. Clin Infect Dis 2001; 32: 1506-1510.

33. Horn B R, Byrd R B. Symulation of pleural disease by disc space infection. Chest 1978; 74: 575-576.

34. Car A J, Crow P G. Vertebral osteomyelitis presenting with abdominal pain and pleural effusion. J R Coll Surg Edinb 1987; 32: 373-374.

35. Sullivan P J, Currie D, Collins J V, Johnstone D J, Morgan A. Vertebral osteomyelitis presenting with pleuritic chest pain and bilateral pleural effusion. Thorax 1992; 47: 395-396.

36. Mateos A, Flórez J, Monte R. Derrame pleural asociado a osteomielitis vertebral. Arch Bronconeumol 1995; 31: 430-431.

37. Shimada T, Nishimura Y, Kimura G, Eto S, Tomita K. Vertebral osteomyelitis presenting with bilateral pleural effusion in a leprous patient. Diagn Microbiol Infect Dis 1996; 24: 101-103

38. Jari S, El-Gamel A, Meadows T, Campbell C. Spinal osteomyelitis presenting with a life-threatening pleural empyema. Spine 1996; 21: 28062808

39. Liesker K R, Taconis W K, Plasmans C M, Schreurs A J. Vertebral ostemyelitis caused by thoracic empyema, or viceversa? Eur Respir J 1996; 9: 2426-2428.

40. Gómez N, Penelas-Cortés Y, Ibáñez J, González M, Sánchez M L. Espondilodiscitis infecciosas en un área sanitaria gallega, 1983-2003. An Med Interna (Madrid) 2004; 21: 533-539.

41. Osenbach R K, Hitchom P W, Menezes A H. Management of pyogenic vertebral osteomyelitis in adults. Surg Neurol 1990; 33: 266-275.

42. Patzakis M J, Rao S, Wilkins J, Moore T M, Harvey PJ. Analysis of 61 cases of vertebral osteomyelitis. Clin Orthop 1991; 269: 142-150.

43. Perronne C, Saba J, Behloul Z, Salmon-Ceron D, Leport C, Vildé J L, Kahn M F. Pyogenic and tuberculous spondylodiskitis (vertebral osteomyelitis) in 80 adult patients. Clin Infect Dis 1994; 19: 746-750

44. Belzunegui J, Del Val N, Intxausti J J, De Dios J R, Queiro R, González C. Vertebral osteomyelitis in northen Spain. Report of 62 cases. Clin Exp Rheumatol 1999; 17: 447-452.

45. Maiuri F, Laconetta G, Gallicchio B, Manto A, Briganti F. Spondylodiscitis. Clinical and magnetic resonance diagnosis. Spine 1997; 22: 17411746. 